\title{
Método variacional aplicado ao estudo de um gás de átomos de Fermi em um estado superfluido aprisionados por uma rede óptica quase periódica
}

Variational method applied to study of a Fermi gas atoms in a superfluid state trapped in a quasiperiodic Optical Lattice

\author{
V.A. Nascimento, C.L. Silva, A.F. Silva, R.C.A. Guimarães, E.S.P. Melo \\ Programa de Pós-Graduação em Saúde e Desenvolvimento na Região Centro-Oeste, Faculdade de Medicina, \\ Universidade Federal de Mato Grosso do Sul, Campo Grande, MS, Brasil
}

Recebido em 15 de março de 2016. Revisado em 4 de maio de 2016. Aceito em 15 de maio de 2016

Neste trabalho, nós consideramos um modelo derivado da densidade de um gás de Fermi em um estado superfluido BCS, aprisionado por uma rede óptica quase periódica unidimensional. Reduzindo a equação em 3D para 1D, construímos famílias de gaps sólitons estáveis (GSs) utilizando aproximações variacionais. No limite linear a aproximação variacional prediz exatamente a posição da banda de Bloch que separam os primeiros gaps. Através da aproximação variacional, mostramos a possibilidade de que a não linearidade efetiva atuando em combinação com o potencial de uma rede quase periódica em uma dimensão, permite o surgimento de gaps sólitons iluminados. Variando a amplitude da rede óptica, analisamos a existência e estabilidade dos gaps sólitons iluminados usando um ansatz gaussiano. Os gaps sólitons são estáveis diante de pequenas variações da rede óptica quase periódica. Este artigo pode ser utilizado como um guia de aprendizagem no estudo de átomos frios; estudantes são incentivados a realizarem os cálculos para outros valores de amplitude da rede óptica.

Palavras-chave: Redes ópticas, Gás de Fermi Degenerado, Aproximação Variacional (AV).

In this work, we consider a model derived from energy density for a Fermi gas in the BardeenCooper-Schrieffer (BCS) superfluid state, trapped in a quasiperiodic one-dimensional optical lattice (OL). Reducing the equation to the 3D to 1D form, we construct families of stable 1D gap solitons (GSs) by the variational approximation (VA). In the linear limit, the VA predicts almost exact positions of narrow Bloch bands that separate the first gaps. Through the variational approximation, we show the possibility that the nonlinearity coefficient acting in a combination with the potential of the quasiperiodic one-dimensional optical lattice, allows one dimensional bright gap solitons to arise. Varying the amplitude of the optical lattice, we analyze the existence and stability of bright gap solitons using a gaussian ansatz. GSs are stable against small variations of the quasiperiodic optical lattice. This paper can be used as a learning guide in the study of cold atoms. Students are encouraged to perform variational calculations for other amplitude values of optical lattices.

Keywords: Optical lattice, Degenerate Fermi Gas, Variational Approximation (VA).

\section{Introdução}

As interpretações sobre a condensação de BoseEinstein podem ser encontradas em livros-textos de mecânica estatística, e em artigos publicados na Revista Brasileira de Ensino de Física [1]. Por outro

*Endereço de correspondência: aragao60@hotmail.com lado, o estudo teórico de gases fermiônicos frios em um estado superfluido aprisionados por lasers são uma minoria, e poucos envolvem campos adicionais de aprisionamento como as redes ópticas e suas propriedades [2]. Neste ponto é importante ressaltar que a falta de materiais didáticos, monografias e teses, criam barreiras que impedem ou dificultam o desempenho de alunos de pós-graduação de ciências 
exatas frente a aprendizagem de novos conceitos científicos, como o comportamento de sistemas gasosos interagentes na presença de um campo de luz.

Nas ementas de alguns cursos de ciências exatas, a abordagem dos princípios variacionais e formalismo lagrangeano são realizados através da mecânica clássica, entretanto, pouco do seu conteúdo é aplicado nos estudos que envolvem a formação de sólitons em meio aquoso, sólitons em sistemas moleculares ou em fibras ópticas [3]. Diante do exposto, este artigo apresenta uma forma viável de contornar essas dificuldades dentro de um contexto didático, servindo, inclusive, como preparação para o aprendizado da mecânica clássica aplicada a sistemas dinâmicos em gases quânticos, de grande importância para o estudo de sistemas de partículas e aplicação em computação quântica [4].

De certa forma, a nossa percepção sobre as interações em sistemas fermiônicos fortemente ou fracamente interagentes ainda é superficial, em partes devido à imposição de uma função de onda antissimétrica para muitos férmions, e, o resultado de suas interações [5]. No que concerne as interações atômicas, investigações teóricas e experimentais nos últimos anos tem se concentrado em estudar átomos frios utilizando redes ópticas, sendo capazes de fornecer novas informações sobre os fenômenos de muitos corpos, como a supercondutividade em alta temperatura [6], e processos de interações envolvendo moléculas fermiônicas ${ }^{23} \mathrm{Na}^{40} \mathrm{~K}$ [7]. Inclui-se dentro desse contexto, os testes experimentais envolvendo as teorias de férmions fortemente interagentes $[5,8]$. Em particular, férmions aprisionados em redes ópticas podem simular a física de elétrons em um sólido cristalino, abrindo as portas para novos fenômenos físicos em materiais, envolvendo principalmente a correlação de elétrons [9].

A partir da interação de feixes de lasers contrapropagantes, surge uma rede óptica, que nada mais é do que uma onda de luz estacionária onde os átomos são aprisionados nos nodos e antinodos pela força de dipolo [10]. Devido a sua forma periódica e efeitos não lineares, a formação de sólitons iluminados, também definidos como gaps sólitons iluminados foram estudados experimentalmente em condensados atômicos de ${ }^{(7)} \mathrm{Li}[11],{ }^{(85)} \mathrm{Rb}[12]$ e em moléculas de ${ }^{(40)} \mathrm{K} \mathrm{e}{ }^{(6)} \mathrm{Li}[13]$.

Neste trabalho nós consideramos um modelo hidrodinâmico de um gás de Fermi em um estado superfluido aprisionado por um potencial de uma rede óptica quase periódica unidimensional. O modelo é baseado em uma equação de parâmetro de ordem (função de onda) derivado da densidade de energia de um superfluido BCS (Bardeen-CooperChrieffer) acoplado fracamente. Ou seja, ao contrário dos gases bosônicos em baixas temperaturas, os gases fermiônicos não sofrem uma transição de fase, apenas passam de um regime clássico para quântico. De fato, como já constatado experimentalmente [14], devido às interações atrativas, e, na presença de uma armadilha magneto óptica, um gás de duas componentes com uma mesma energia de Fermi passa para um sistema superfluido.

Utilizando aproximação variacional (AV) para resolvermos a equação hidrodinâmica que descreve o sistema de gases fermiônicos aprisionados, propomos e exploramos uma nova possibilidade de que a não linearidade efetiva para férmions atuando em combinação com um potencial da rede óptica quase periódica em 1D permite o surgimento de gap sólitons fundamentais unidimensionais. Estudos utilizando métodos variacionais e numéricos obtiveram sucesso em demonstrar que gases fermiônicos aprisionados por uma rede óptica simples permitem o surgimento de gap sólitons estáveis [15], assim como métodos quânticos e numéricos estudaram e destacaram as propriedades de energia e momento de gases bosônicos aprisionados por uma rede quase periódica unidimensional [16]. A eficiência de uma rede quase periódica em confinar átomos foi testada através da variação do número de lasers de aprisionamento, ou seja, as evidências experimentais utilizando uma rede quase periódica em 3D criada pela interferência de quatro, cinco ou seis lasers, exploraram a temperatura e expansão balística dos átomos aprisionados, demonstrando que seis feixes de lasers são mais eficientes para capturar átomos [17]. De certa forma, várias propriedades físicas foram exploradas utilizando gases bosônicos aprisionados por redes ópticas simples ou com dupla periodicidade, entretanto, o estudo de gases fermiônicos aprisionados por uma rede óptica quase periódica utilizando métodos variacionais é inovador, uma vez que podem fornecer novas informações sobre a estabilidade de sistemas fermiônicos. Diante do exposto, procuraremos por soluções variacionais para a equação hidrodinâmica utilizando um ansatz (função teste) Gaussiano $\phi(x)$ unidimensional.

O trabalho está organizado como segue. Na seção 2 faremos uma breve introdução teórica sobre sólitons, 
e posteriormente abordaremos os princípios físicos envolvidos na obtenção de uma rede óptica quase periódica e formação de gaps sólitons iluminados. $\mathrm{Na}$ seção 3 nós apresentaremos o modelo hidrodinâmico proposto e procuraremos por gaps sólitons iluminados estáveis em 1D por meio da aproximação variacional para três valores de amplitudes de rede. Na seção 4, apresentamos os resultados obtidos como consequência da aproximação variacional para gaps sólitons iluminados em 1D. A seção 5 consta com a conclusão dos resultados obtidos e algumas sugestões para trabalhos futuros, como também incentivamos ao leitor realizar os cálculos variacionais para outros valores de amplitudes da rede óptica quase periódica para estudar a estabilidade dos gaps sólitons formados.

\section{Sólitons e o potencial de uma rede óptica unidimensional}

John Scott Russel em 1834 foi um dos pioneiros em estudar qualitativamente a propagação de ondas aquáticas no canal de Edinburgo-Glasgow. Posteriormente em 1895 a dedução matemática de tais fenômenos aquáticos foram obtidas por D.J. Korteweg e G. de Vries. Porém, foi em 1965 que Zabusky e M. Kruskal estudando as soluções de D.J. Korteweg e G. de Vries definiram tal efeito como sólitons ou ondas solitárias, onde on denomina partículas, como fótons, elétrons e etc [Ref.3, B.A. Malomed, 2006]. Os sólitons correspondem as soluções particulares de algumas equações não lineares, descrevendo a propagação de excitações em meios contínuos envolvendo efeitos de dispersão e não linearidade. Geralmente existem dois tipos de sólitons: os sólitons iluminados ou brilhantes, que são pacotes de ondas localizados que não perdem a sua forma durante a propagação, isto é, a sua amplitude de distribuição $\left(|\phi(\mathrm{x}, \mathrm{t})|^{2}\right)$ mantem-se constante durante um longo tempo de evolução temporal (T) e espacial (X) (Fig.1 (a)); e os escuros, que são buracos se movendo em um fundo de matéria (Fig.1 (b)), neste caso, a sua amplitude de distribuição também mantem-se constante durante uma evolução temporal e espacial. Os dois exemplos de solitons apresentados na Fig.1 são meramente ilustrativos, entretanto, a função de distribuição $\left(|\phi(\mathrm{x}, \mathrm{t})|^{2}\right)$ pode ser a solução de uma equação não linear do tipo Schrödinger aplicada a sistemas gasosos ou fibras ópticas. Em nosso trabalho, inicialmente consideraremos na próxima seção uma equação não linear dependente do tempo tridimensional, entretanto, mediante a imposição de algumas considerações físicas, a referida equação em 3D será transformada em uma equação unidimensional e independente do tempo.

Dependendo do fenômeno e das condições físicas, os sólitons são denominados de gaps sólitons. Na física do estado sólido, denominados de gaps a descontinuidade no espectro de energia de elétrons livres em um cristal sujeito a um potencial periódico, ou seja, regiões espectrais onde não existe a propagação de ondas. Entretanto, quando a não linearidade permite a localização de ondas dentro de uma banda de gaps linear, formam-se gaps sólitons iluminados. Os gaps sólitons, estão presentes em outros fenômenos como em fibras ópticas e guias de ondas, sendo a interação de dispersão cromática (domínio temporal) ou difração (sólitons espaciais) de ondas eletromagnéticas e não linearidade cúbica, responsáveis pelo seu surgimento.

A equação de Schrödinger com um termo não linear cúbico na presença de um potencial óptico periódico possui soluções do tipo sólitons iluminados quando as interações atômicas são atrativas,
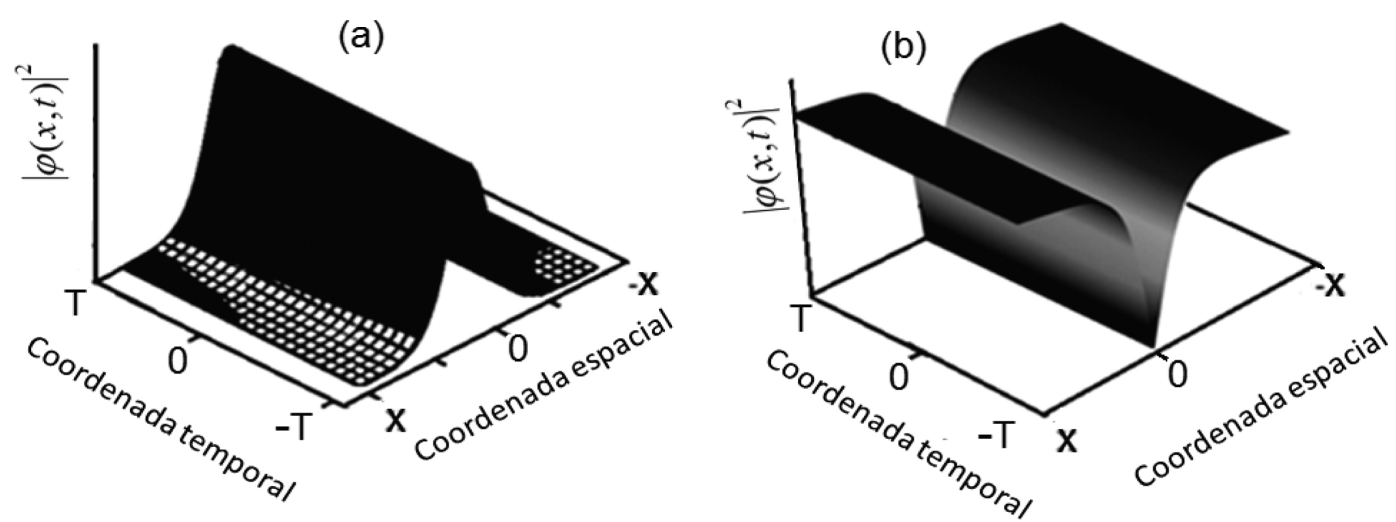

Figura 1: Exemplo ilustrativo de um sóliton iluminado (a) e um sóliton escuro (b). 
e quando as interações são repulsivas, as soluções são sólitons escuros. Experimentalmente, um potencial periódico unidimensional de aprisionamento de átomos frios é obtido utilizando a interação de dois lasers, neste caso, similar ao que ocorre na física do estado sólido, funções de ondas localizadas tomam a forma de sólitons iluminados dentro de gaps proibidos do espectro da banda de gap linear, sendo também denominados de gaps sólitons iluminados [2].

Um dos mais comuns potenciais de aprisionamento de dipolo é a rede óptica. Obtêm-se uma rede óptica quase periódica unidimensional a partir da interação de dois feixes de lasers contra - propagantes sintonizados com a mesma frequência $\left(\omega_{1} \mathrm{e}\right.$ $\omega_{2}$ ) e intensidades. Entretanto, ambos os lasers possuem polarizações circulares opostas e uma pequena diferença de fase $[16,18]$. Um padrão de interferência é produzido devido à interação, restringindo o movimento os átomos nos nodos e antinodos pela força de dipolo. Para ser mais preciso, o campo elétrico oscilante do laser induz um momento de dipolo no átomo, o qual então interage com o campo de luz. Deste modo, um potencial de aprisionamento é criado e os átomos são aprisionados. A Fig.2 ilustra o processo de interação entre dois lasers.

Os dois feixes de lasers possuindo diferentes números de ondas $K_{L 1}$ e $K_{L 2}$ geram potenciais periódicos ao longo de x. A partir da Fig.2 observamos que $K_{L 2}$ varia com o ângulo $\theta$, desde que $K_{L 2}=K_{\text {Laser }} \cos \theta$, e $d$ trata-se do espaçamento da rede. Após algumas manipulações matemáticas conforme as Refs. [16, 18], o potencial da rede quase periódica pode ser escrito como:

$$
V(x)=\varepsilon_{1}\left(\cos \left(2 K_{L 1} x+\phi\right)\right)+\varepsilon_{2}\left(\cos \left(2 K_{L 2} x\right)\right)
$$

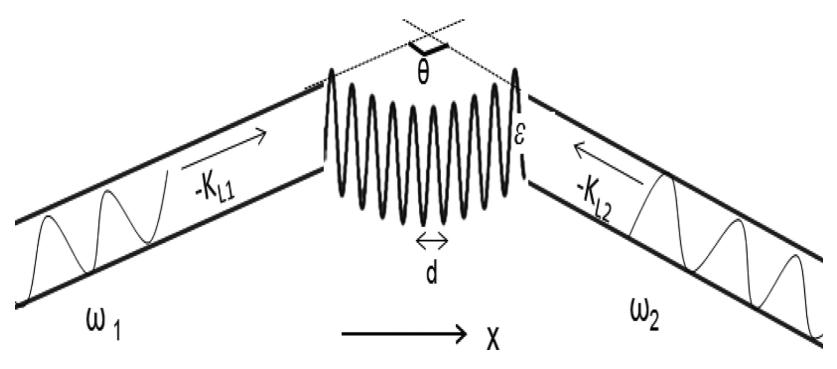

Figura 2: Rede óptica unidimensional criada pela intersecção de dois feixes de lasers na direção $x$, com vetores de ondas $K_{L 1}$ e $K_{L 2}$, e frequências $\omega_{1}$ e $\omega_{2}$. O parâmetro $\varepsilon$ é a profundidade ou amplitude e $d$ espaçamento da rede, $\theta$ refere-se ao ângulo de intersecção dos lasers. onde $\phi$ refere-se à fase relativa entre as duas ondas estacionárias, $\varepsilon_{1}$ e $\varepsilon_{2}$ são as amplitudes ou profundidades da rede, podendo ser ajustadas experimentalmente variando a intensidade dos lasers. Teoricamente ao considerarmos $K_{L 1}=1$ e $\alpha=K_{L 2} / K_{L 1}$ o potencial descrito na Eq.(1) torna-se

$$
V(x)=\varepsilon_{1}(\cos (x+\phi))+\varepsilon_{2}(\cos (\alpha x))
$$

Se $\varepsilon_{1}=\varepsilon_{2}=\varepsilon$, a Eq.(2) transforma-se em:

$$
V(x)=\varepsilon[\cos (x+\phi)+\cos (\alpha x)] .
$$

Um exemplo da forma de um potencial de rede óptica quase periódica que estudaremos nesse trabalho está exposto na Fig. 3, onde adotamos $\phi=1$ e $\alpha=0.5$ [16], e consideramos que ambos os cosenos possuem a mesma amplitude $\varepsilon_{1}=\varepsilon_{2}=\varepsilon=1$. Desta forma, a partir da Eq. (3), ao variarmos a amplitude $\varepsilon$ podemos obter outros tipos de redes quase periódicas que também serão explorados nesse trabalho nas próximas seções. A Eq.(3) não envolve a parte temporal.

\section{O modelo teórico considerado}

Nesta secção, a partir da densidade Lagrangeana, obteremos a equação Euler-Lagrange. Para obtermos um Fermi superfluido, é necessário considerarmos duas partículas (átomos alcalinos em dois estados hiperfinos diferentes) com spins $1 / 2$ e massas $m$, aprisionados pelo potencial do laser e um campo magnético, que faz com que os férmions emparelhemse, similar aos pares de elétrons que produzem a supercondutividade, conhecidos como pares de Cooper. Embora os férmions não possuam cargas para formar a supercondutividade, ao estarem em pares implica em uma interação, ou seja, o emparelhamento de onda - s $(l=0)$ entre duas componentes do gás atômico acarreta em uma superfluidez. Como realizado nos estudos teóricos [15], átomos fermiônicos formam um superfluido BCS de pares de Cooper devido à interação fraca entre férmions com spins de orientações opostas, desta forma, para obtermos uma equação efetiva para o parâmetro de ordem do superfluido basta lembrar que em $3 \mathrm{D}$ a densidade de energia de um superfluido é dada pela expressão [19]

$$
\varepsilon_{3 D}=(3 / 5) \rho_{3 D} \varepsilon_{F}
$$

onde $\varepsilon_{F}=\left(\hbar k_{F}\right)^{2} /(2 m)$ é a energia de Fermi, $\hbar k_{F}$ é o momento de Fermi e $\rho_{3 D}$ é a densidade atômica, 


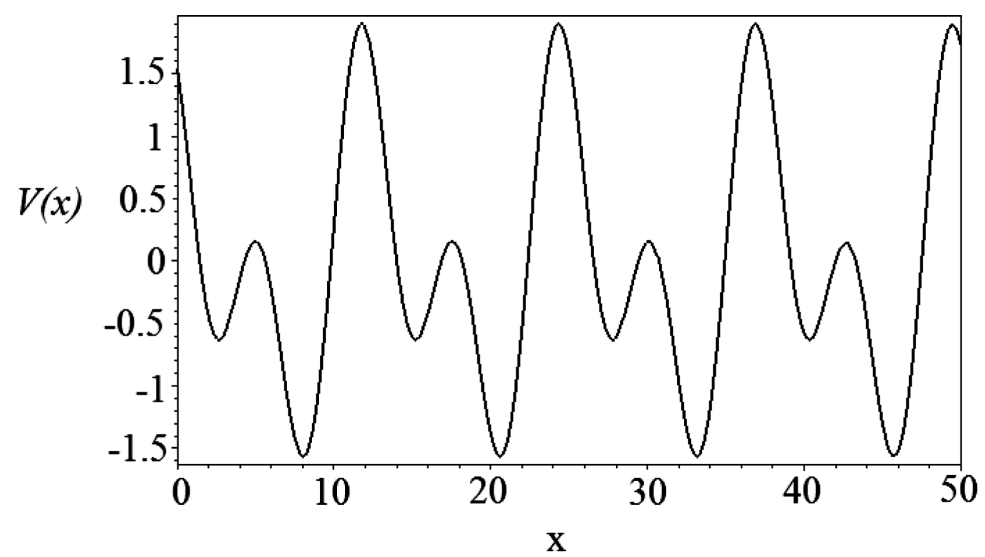

Figura 3: Potencial de uma rede óptica quase periódica unidimensional obtida a partir da Eq. (2) considerando $\varepsilon=1$, $\phi=1$ e $\alpha=0.5$

levando em consideração a paridade de Cooper, a densidade total de átomos é escrita como

$$
\rho_{3 D}=\frac{2}{(2 \pi)^{3}} \int_{0}^{k_{F}} 4 \pi k^{2 d} k \equiv \frac{1}{3 \pi^{2}}\left(\frac{2 m \varepsilon_{F}}{\hbar^{2}}\right)^{3 / 2}
$$

A partir da Eq.(5), a densidade de energia representada pela Eq.(4) torna-se.

$$
\varepsilon_{3 D}=\frac{3(3 \pi)^{2 / 3}}{10 m} \rho_{3 D}^{5 / 3}
$$

De acordo com a teoria de Ginzburg-Landau, todas as características e propriedades do fenômeno da supercondutividade provém de uma função termodinâmica chamada densidade de energia livre [20]. A partir dessa consideração, o superfluido BCS é descrito por um parâmetro de ordem complexo $\psi(r, t)$, no qual $\rho_{3 D} \equiv|\psi(r, t)|^{2}$. Ou seja, $\psi(r, t)$ está relacionada diretamente com a densidade atômica, quanto maior o seu valor, maior será a densidade atômica e consequentemente maior será o efeito de superfluidez. A equação de evolução para $\psi(r, t)$ pode ser obtida a partir da correspondente densidade lagrangeana,

$$
\begin{aligned}
& L=\frac{i \hbar}{2}\left(\psi^{*}(r, t) \frac{\partial \psi(r, t)}{\partial t}-\psi(r, t) \frac{\partial \psi^{*}(r, t)}{\partial t}\right) \\
& -\left(\frac{\hbar^{2}}{2 m_{e f}}\right)|\nabla \psi(r, t)|^{2}-V(r)|\psi(r, t)|^{2} \\
& -\varepsilon_{3 D}
\end{aligned}
$$

que ao substituirmos a Eq.(6) na Eq.(7), e levarmos em consideração $\rho_{3 D} \equiv|\psi(r, t)|^{2}$, obtemos a equação de Euler-Lagrange

$$
\begin{aligned}
& i \hbar \frac{\partial \psi(r, t)}{\partial t}=\frac{\hbar^{2}}{2 m_{e f}}\left[-\nabla^{2}+\left(3 \pi^{3}\right)^{2 / 3}\right. \\
& \left.\times|\psi(r, t)|^{4 / 3}\right] \psi+V(r) \psi(r, t)
\end{aligned}
$$

onde $m_{e f f} \approx 2 m$ é a massa efetiva associada ao parâmetro de ordem do superfluido, $V(r)$ é o potencial de aprisionamento, $\nabla^{2}$ é o Laplaciano em 3D (operador energia cinética). A Eq.(8) está sujeita à condição de normalização $N=\int\left|\psi(r, t)^{2}\right| d x d y d z$ e estados estacionários podem ser obtidos ao considerarmos $\psi(r, t)=\phi(r) \exp (-i \mu t / \hbar)$, onde $\mu$ é o potencial químico e $\phi(r)$ é uma função real que satisfaz a equação,

$$
\begin{aligned}
& \mu \phi(r)=\frac{\hbar^{2}}{2 m_{e f}}\left[-\nabla^{2}+\left(3 \pi^{2}\right)^{2 / 3} \phi^{4 / 3}(r)\right] \phi(r) \\
& +V(r) \phi(r)
\end{aligned}
$$

Em alguns trabalhos teóricos, é comum além de considerarmos o potencial da rede óptica $(V(x))$ acrescentarmos o potencial de confinamento harmônico extra de uma armadilha óptica ou magnética $\left(V_{A M}\right.$ $(y, z))$, ou seja, o potencial de aprisionamento tridimensional passa a ser escrito como $V(r)=$ $V_{A M}(y, z)+V(x)$, onde o primeiro termo $V_{A M}(y, z)=$ $(1 / 2) m \omega_{\perp}^{2}\left(y^{2}+z^{2}\right)$ refere-se ao potencial extra do tipo oscilador harmônico em 2D (na forma de charuto) que representa o potencial de aprisionamento magnético experimental, sendo $m$ a massa atômica e $\omega_{\perp}$ é a frequência do laser de aprisionamento transversal (ou frequência do confinamento harmônico), e o segundo termo $V(x)$ trata-se do potencial da rede óptica descrito pela Eq.3. 
Assumiremos nesse trabalho, que o potencial de aprisionamento harmônico extra do tipo charuto caracterizado pela frequência de aprisionamento $\omega_{\perp}$ ao longo da direção axial é fraco, isso equivale que seu efeito sobre os estados estacionários do condensado na rede óptica é pequeno. Mediante tais considerações o potencial de aprisionamento extra é desprezado nos cálculos, e a Eq.(9) pode ser reduzida para o caso unidimensional (como considerado nas Refs. $[2,21])$. É interessante enfatizarmos ao leitor que mesmo na presença de um potencial extra do tipo charuto, a Eq.(8) e (9), podem ser reduzidas para o caso unidimensional sob a condição de que as distâncias entre os níveis de energias no potencial transverso $\left(\hbar \omega_{\perp}\right)$ é muito menor que a energia de Fermi $\left(\varepsilon_{F}\right)$. Isso implica que a energia de Fermi corresponde a um estado excitado do potencial de confinamento transverso.

\section{Aproximação variacional para sólitons em um gás de férmions em uma dimensão.}

A partir das considerações impostas na secção 2 e 3 , a Eq. (9) em três dimensões pode ser transformada em uma equação unidimensional ao considerarmos a relação

$$
\begin{aligned}
& \phi(x, y, z)=\Phi(x) \Phi(y, z) \\
& =\Phi(x) \exp \left(-\left(y^{2}+z^{2}\right) / 2 a_{h o}^{2}\right),
\end{aligned}
$$

onde o segundo termo da Eq. (10) aproxima-se dos estados excitados do oscilador harmônico transversal, sendo $a_{h o}=\sqrt{\hbar /\left(m \omega_{\perp}\right)}$ o tamanho do potencial de aprisionamento harmônico oscilatório, e $\omega_{\perp}$ é a respectiva frequência harmônica.

A substituição da Eq. 10 e a inserção do potencial da rede óptica quase periódica (Eq.(3)) na Eq. (9), após multiplicação por $\Phi(y, z)$ e integração em relação à $y$ e $z$, produz uma equação de EulerLagrange em 1D para férmions aprisionados por um potencial da óptica quase periódica unidimensional escrita como segue,

$$
\begin{aligned}
& \mu \Phi(x)=-\frac{\hbar^{2}}{2 m_{e f}} \frac{d^{2} \Phi(x)}{d x^{2}} \\
& +\left(\frac{3 \pi^{2}}{2}\right)^{2 / 3} \frac{3 \hbar^{2}}{10 m_{e f}} \times(\Phi(x))^{7 / 3} \\
& -\varepsilon[\cos (x+\phi)+\cos (\alpha x)] \Phi(x)
\end{aligned}
$$

Na Eq. (9) existe um conjunto de constantes descritas em um sistema de coordenadas tridimensionais, e que ao passarmos para o caso unidimensional se transforma em outro conjunto no novo sistema. Diante da problemática, devemos reescrever as relações entre as novas e antigas constantes. Para isso, devemos identificar as dimensões das variáveis envolvidas no problema, como a massa efetiva $\left(m_{e f}\right)$, o espaço $(L)$ e constantes significativas como exemplo $\omega^{2} \mathrm{e}$ $\mu$. A Eq. (11), por sua vez pode ser escalonada assumindo,

$$
\begin{aligned}
& \Phi(x) \equiv \sqrt{\frac{2 N}{\lambda}} a_{o h}^{-1} \tilde{\phi}(x), \\
& \mu \equiv{\frac{\hbar^{2}}{m}}_{\text {ef }}\left(\frac{2 \pi}{\lambda}\right)^{2} \tilde{\mu} \quad \text { e } \quad x \equiv \frac{\lambda}{2 \pi} \tilde{x} .
\end{aligned}
$$

Que após escalonamento passa a ser escrita como

$$
\begin{aligned}
& \mu \phi(x)=-\frac{1}{2} \frac{d^{2} \phi(x)}{d x^{2}}+g_{1 D}(\phi(x))^{7 / 3} \\
& -V_{0}[\cos (x+\phi)+\cos (\alpha x)] \phi(x) .
\end{aligned}
$$

A partir da Eq.12 escalonada obtemos alguns parâmetros importantes no nosso estudo, como a quantidade física $g_{1 D}=\left(3 m_{e f} / 10 m\right)\left(3 \lambda^{2} N / 8 \pi a_{o h}^{2}\right)^{2 / 3}$ denominada de força efetiva de não linearidade para férmions em $1 \mathrm{D}$, lembrando que $a_{h o}^{2}=\hbar / m \omega_{\perp}$ tratase do comprimento do oscilador harmônico, $N$ tratase do número de átomos, $\lambda$ é o comprimento de onda do laser. A variável $V_{0} \equiv \varepsilon m_{e f}(\lambda / 2 \pi \hbar)^{2}$ é proporcional à amplitude da rede $\varepsilon$. Ou seja, ao variarmos $V_{0}$ ao longo do nosso trabalho, estamos nos referindo à variação de $\varepsilon$. A Eq.12 é utilizada para estudar férmions e inclui um termo não linear repulsivo $|\phi(x)|^{4 / 3} \phi(x)[15]$. Este termo não considera as colisões entre átomos, porém é indiretamente induzido pelo princípio de Pauli através da distribuição de Fermi dos átomos. A Eq. 12 está sujeita à normalização $\int|\phi(x)|^{2} d x=1$.

As soluções para a Eq. (12) são obtidas como segue; primeiramente assumiremos no decorrer desse trabalho que $\phi=1$ e $\alpha=0.5$, posteriormente, consideraremos que soluções variacionais são obtidas ao utilizarmos um ansatz (função teste) variacional para sólitons em 1D na forma Gaussiana dada por [22],

$$
\phi(x)=\frac{\sqrt{M}}{\pi^{1 / 4} W^{1 / 2}} \exp \left(-\frac{x^{2}}{2 W^{2}}\right)
$$


sendo $M$ (norma) e $W$ (largura) os parâmetros variacionais do sóliton em 1D. De acordo com Vitor M. Perez Garcia [22], a ideia de utilizarmos o método variacional e considerarmos uma função teste (ansatz gaussiano) com uma forma fixa e alguns parâmetros livres é que podemos reduzir um problema de equações diferenciais parciais, a uma equação diferencial ordinária de segunda ordem tipo Newton. A escolha desse tipo de função de onda é justificável primeiramente em decorrência de o ansatz gaussiano ser utilizado como solução em vários estudos de condensados bosônicos e fermiônicos $[2,15]$, inclusive na Ref. [22] foram deduzidas as equações para quatorze parâmetros da gaussiana, parâmetros estes que predizem resultados como colapsos, largura da nuvem, estabilidade e número de átomos em condensados e etc. A segunda justificativa é que os resultados (soluções) obtidos utilizando ansatz gaussiano são próximos aos resultados numéricos $[15,22,23]$, desta forma, ansatz gaussianos e métodos numéricos são equivalentes em prever o comportamento de sólitons.

Para encontrarmos as equações que fornecem a evolução de todos os parâmetros variacionais, devemos calcular a lagrangeana efetiva através da densidade lagrangeana no espaço de coordenadas utilizando a relação.

$$
L_{D . e f}=\left\langle L_{D}\right\rangle=\int_{-\infty}^{\infty}\left[L_{D}\right] d x
$$

A partir da substituição do anzatz (Eq. (13)) na lagrangeana (Eq. (12)) e realização dos cálculos impostos pela Eq. (14), obtemos uma lagrangeana efetiva para férmions aprisionados por uma rede óptica quase periódica, escrita em termos dos parâmetros da função de onda da gaussiana,

$$
\begin{aligned}
& L_{e f}=\mu_{F}(M-1)-\frac{M}{4 W^{2}}-\left(\frac{3}{5}\right)^{5 / 2} \frac{g_{1 D} M^{5 / 3}}{\pi^{1 / 3}(W)^{2 / 3}} \\
& +V_{0}\left(e^{-0.062 V^{2}}+0.54 e^{-0.25 V^{2}}\right) M
\end{aligned}
$$

A seguir, as equações de evolução dos parâmetros $\mu, g_{1 D}, M$ e $W$ explícitos na Eq. (15), são obtidas ao resolvermos as equações de Euler-Lagrange

$$
\frac{d}{d t}\left(\frac{\partial L_{e f}}{\partial \dot{q}_{j}}\right)-\frac{\partial L_{e f}}{\partial q_{j}}=0,
$$

onde adotamos a notação representada por $q_{j}=\{\mu$, $M, W\}$.
A primeira equação variacional é obtida a partir da lagrangeana efetiva (Eq. (15)), assim, $\partial L_{e f} / \partial \mu_{D}=$ 0 produz como esperado $M=1$, que será substituído nas outras equações variacionais abaixo. Desenvolvendo os cálculos $\partial L_{e f} / \partial W=0$, teremos a relação entre a largura do sóliton $(W)$ e o coeficiente de não linearidade $\left(g_{1 D}\right)$ conforme explícito abaixo,

$$
\begin{aligned}
& 1+\frac{4 g_{1 D} W^{4 / 3}}{5 \pi^{1 / 3}}-0.248 V_{0} W^{4} e^{-0.062 W^{2}} \\
& =0.54 V_{0} W^{4} e^{-0.25 W^{2}}
\end{aligned}
$$

Considerando $\partial L_{e f} / \partial M=0$ obtemos $\mu$ (potencial químico) em função da largura do sóliton $(W)$ e do coeficiente de não linearidade para férmions $\left(g_{1 D}\right)$ :

$$
\begin{aligned}
& \frac{1}{4 W^{2}}+\frac{g_{1 D}}{\pi^{1 / 3}(W)^{2 / 3}}-V_{0} e^{-0.062 W^{2}} \\
& -0.54 V_{0} e^{-0.25 W^{2}}=\mu .
\end{aligned}
$$

As soluções das Eqs. (17) e (18) produzem uma dependência do coeficiente de não linearidade $\left(g_{1 D}\right) \mathrm{em}$ função do potencial químico $\left(\mu_{D}\right)$ para gaps sólitons iluminados em 1D. Estando de posse da expressão da não linearidade em função do potencial químico, devemos procurar o valor de $W$ que minimiza esta expressão. Os dois sistemas de Eq. (17) e (18) são equivalentes; nesse caso, toda solução de qualquer um dos sistemas também é solução do outro. Uma solução de um sistema é uma sequência de números (valores dos parâmetros variacionais) que satisfaz as equações simultâneas. Uma alternativa para resolvermos um sistema de equações lineares é utilizarmos o método de Gauss-Seidel (método de iteração), ou outros métodos abordados extensamente e disponíveis em livros-texto de física computacional e métodos numéricos.

Para averiguarmos a estabilidade dos gap sólitons formados, variamos nas Eq.(17) e (18) a amplitude da rede quase periódica, considerando inicialmente três casos em estudo, $V_{0}=1,5$ e 10 , mantendo parâmetros como $\phi=1$ e $\alpha=0.5$ fixos. A próxima secção destaca os resultados obtidos a partir de tais considerações.

\section{Resultados obtidos a partir da aproximação variacional.}

Ao resolvermos as equações variacionais (Eq. (17) - 18), obtemos a força efetiva de não linearidade 
$\left(g_{1 D}\right)$ para férmions em função do potencial químico $(\mu)$ para o caso unidimensional (Fig.4). É interessante destacarmos que em um limite linear, quando $g_{1 D}=0$, a solução variacional prediz os valores para o potencial químico, o qual coincide (equivale) ao valor (localização) da primeira borda esquerda na primeira banda de gap; para a Fig.4(a) temos $\mu\left(V_{0}=1\right) \approx-1.12$; Fig.4(b): $\mu\left(V_{0}=5\right) \approx-6.73 \mathrm{e}$ na Fig.4(c): $\mu\left(V_{0}=10\right) \approx-14.02$. Procedimentos similares para encontrar as primeiras bandas foram realizados nas Refs. [2,15]. Na Fig.4, as barras verticais representam as bandas de Bloch que separam os gaps. Outros cálculos foram realizados variando os valores de $g_{1 D}$ e $V_{0}$ conforme veremos ao longo do texto.

As famílias de gap sólitons fundamentais para um gás de férmions são encontradas na primeira banda de gap do potencial periódico $V(x)=V_{0}[\cos (x+$ $\phi)+\cos (\alpha x)]$ conforme Fig.4. Embora as soluções variacionais predizerem famílias de gap sólitons fundamentais na primeira banda de gap e continuam através da segunda banda de gap, elas não sentem a presença das bandas de Bloch que separam as banda de gap, comportamento este, similar ao encontrado por outros tipos de rede ópticas $[14,16]$.

Os respectivos aumentos de $g_{1 D}(\mu)$ podem ser observados à medida que nos afastamos da borda da primeira banda de gap (Veja Fig. 4), ou seja, é evidente que famílias de gaps sólitons iluminados em gases fermiônicos originam do fundo da banda de gap. Isto é, não linearidade fraca (pequenos valores para $N$ ), acarreta em estados de gaps sólitons iluminados que surgem próximos das bordas inferiores das bandas de gap linear. Como o potencial químico está aumentando, o número de átomos no gap também aumenta. Os gaps sólitons iluminados em condensados repulsivos surgem do fundo da banda devido à instabilidade modulacional de ondas de Bloch [24], o mesmo ocorre para o gás de Fermi aprisionado por uma rede óptica quase periódica unidimensional.

Famílias de gaps sólitons iluminados são localizadas em um único sítio da rede óptica para pequenos valores do coeficiente de não linearidade $[2,15,24,25]$. Em vista disso, é possível obtermos vários tipos de gaps sólitons estáveis com distintos valores de $\mu$ próximos das bordas das bandas de gap. Como na Fig. 4 existem várias famílias de gaps sólitons iluminados, podemos observar as suas formas utilizando a gaussiana dada pela Eq. (13) que possui largura $(W)$, no qual seus valores são obtidos através da solução da Eq. (17). A Fig. 5 mostra nove exemplos de gaps sólitons iluminados pertencentes à primeira banda de gap do espectro em 1D, contendo os valores das amplitudes da rede óptica quase periódica, coeficientes de não linearidades e potencias químicos.

Na Fig.5, analisando os parâmetros obtidos ao considerarmos a amplitude da rede quase periódica $V_{0}=1$, obtemos; $\mu=-0.81\left(g_{1 D}=0.5\right), \mu=-0.53$ $\left(g_{1 D}=1\right)$ e $\mu=-0.28\left(g_{1 D}=1.5\right)$. Para a amplitude de rede $V_{0}=5 ; \mu=-6.3\left(g_{1 D}=0.5\right)$, $\mu=-5.9\left(g_{1 D}=1\right)$ e $\mu=-5.5\left(g_{1 D}=1.5\right)$. Ao consideramos a amplitude da rede $V_{0}=10$, te$\operatorname{mos} ; \mu=-13.5\left(g_{1 D}=0.5\right), \mu=-13\left(g_{1 D}=1\right) \mathrm{e}$ $\mu=-12.6\left(g_{1 D}=1.5\right)$. Os três valores de amplitudes considerados revelam que existe um aumento significativo nos valores do potencial químico e não
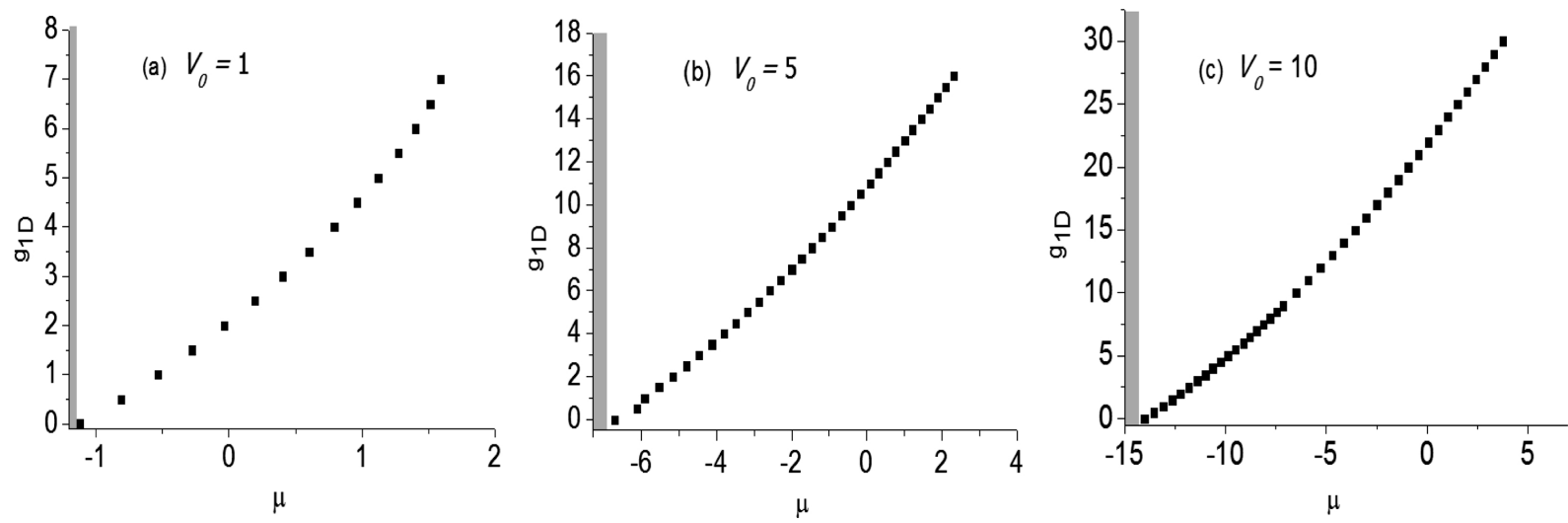

Figura 4: Resultados variacionais para o coeficiente de não linearidade efetiva $g_{1 D}$ para férmions versus o potencial químico $\mu$ em 1D (Eq.17). As linhas pontilhadas são obtidas para as amplitudes: (a) $V_{0}=1$; (b) $V_{0}=5$ e (b) $V_{0}=$ 10. Valores dos potenciais químicos para a família de gap sóliton na primeira banda de gap do potencial quase periódico $V(x)=\varepsilon[\cos (x+\phi)+\cos (\alpha x)]$. As barras verticais representam as bandas de Bloch que separam os gaps, obtidas quando $g_{1 D}=0$. 

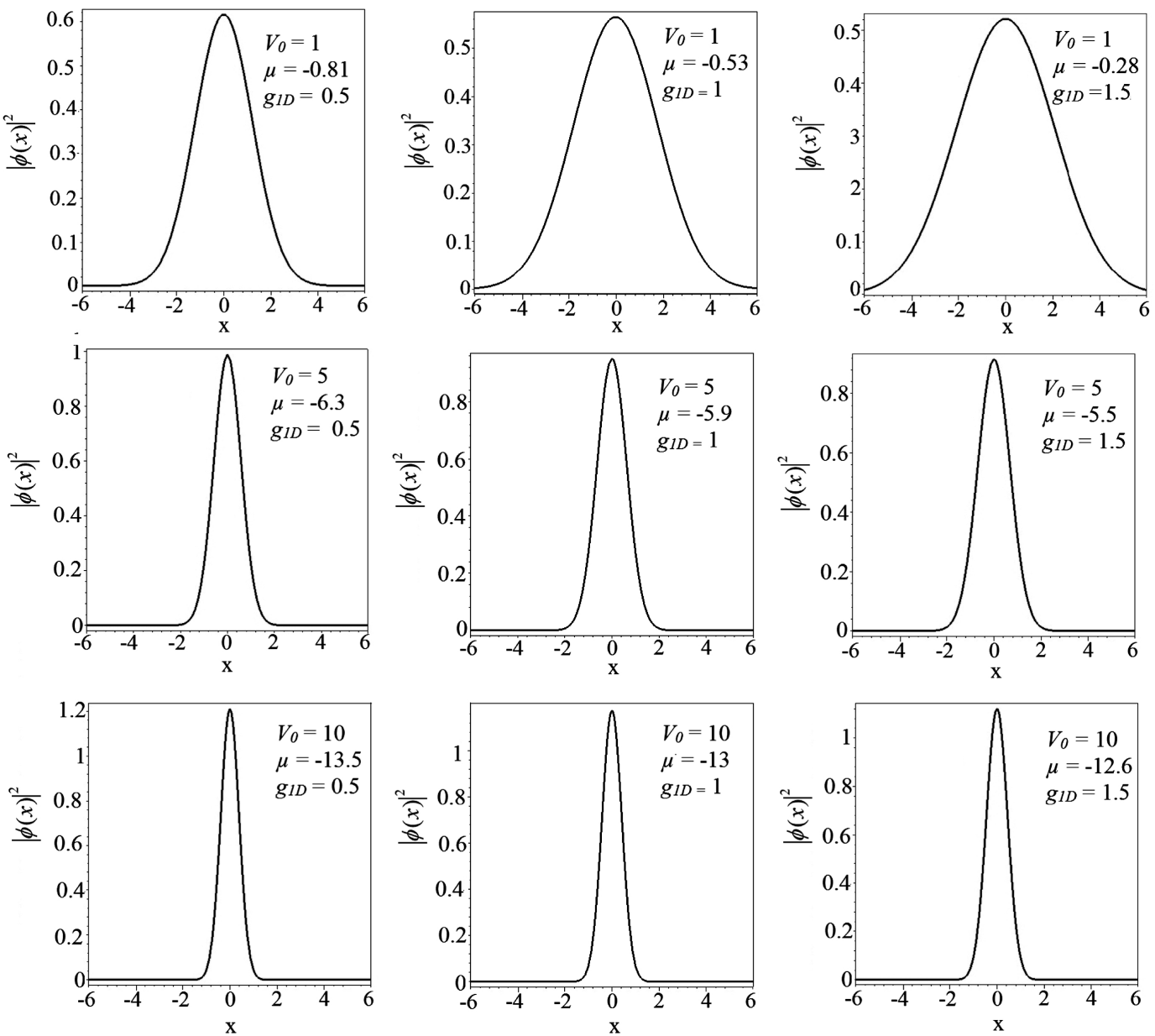

Figura 5: Formas típicas de gap sólitons fundamentais para um gás de férmions em 1D. Exemplo de nove picos estáveis para gaps sólitons na forma Gaussiana obtidos através da Eq. (13) e pertencente a primeira banda de gap do espectro da Fig.4. Nos gráficos constam os valores da amplitude das redes óptica $\left(V_{0}=1,5\right.$ e 10), os coeficientes de não linearidades $\left(g_{1 D}\right)$ e potenciais químicos $(\mu)$ para o caso de uma rede quase periódica.

linearidade. Nesses casos, quando analisamos os comportamentos das gaussianas, observamos pequenos aumentos nas larguras e uma diminuição de sua amplitude à medida que ocorre uma variação crescente nos valores de não linearidade e potencial químico.

Os resultados apresentados na Fig. 5 podem ser interpretados de outra forma, através da Fig. 6 que destaca o comportamento do potencial químico $(\mu)$ versus a variação da amplitude da rede óptica (onde consideramos: $1 \leq V_{0} \leq 10$ ), revelando que, com o aumento da amplitude da rede óptica quase periódica, o potencial químico torna-se cada vez mais negativo. Ou seja, ao observamos a Fig.5 ou Fig.6, e considerarmos os resultados contendo apenas os valores para a não linearidade $g_{1 D}=0.5$; obte$\operatorname{mos} \mu\left(V_{0}=1\right) \approx-0.81$, e $\mu\left(V_{0}=5\right) \approx-6.3 \mathrm{e}$ $\mu\left(V_{0}=10\right) \approx-13.5$. Quando consideramos a não linearidade $g_{1 D}=1$; temos $\mu\left(V_{0}=1\right) \approx-0.53$,
$\mu\left(V_{0}=5\right) \approx-5.9$, e $\mu\left(V_{0}=10\right) \approx-13$. E para a não linearidade $g_{1 D}=1.5 ; \mu\left(V_{0}=1\right) \approx-0.28$, $\mu\left(V_{0}=5\right) \approx-5.5, \mu\left(V_{0}=10\right) \approx-12.6$. Como podemos observar na Fig. 5, a gaussiana diminui a sua largura e sofre um aumento de sua amplitude, evidenciando que gaps solitons são estáveis para valores próximos a borda da banda, mesmo quando ocorre uma pequena variação da amplitude da rede quase periódica. Um comportamento similar ao obtido na Fig.6, também foi observado nos resultados obtidos para um gás de férmions aprisionados em uma rede óptica simples unidimensional $[2,15]$.

Os resultados obtidos através do ansatz Gaussiano utilizando aproximações variacionais confirmam a existência de gaps sólitons fundamentais, que surgem devido a atuação da não linearidade efetiva para férmions em combinação com um potencial da rede óptica quase periódica em 1D. O compor- 


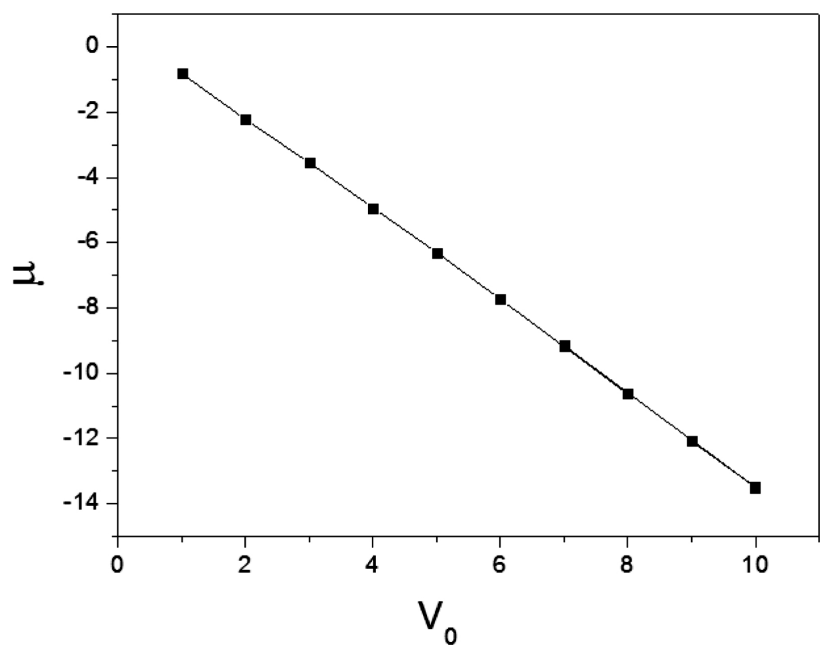

Figura 6: Potencial químico $(\mu)$ versus amplitude da rede óptica $\left(V_{0}\right)$ quase periódica. Consideramos a amplitude da rede óptica dentro do intervalo: $1 \leq V_{0} \leq 10$

tamento das gaussianas (Fig.5) reflete de maneira eficaz a estabilidade dos gaps sólitons iluminados (Fig.4), diante das variações da amplitude da rede óptica quase periódica (Fig.6) e da não linearidade. $\mathrm{Na}$ literatura existem trabalhos que estudam a dependência de $N$ (norma, proporcional ao número de átomos) em função do potencial químico [23,24], potencial químico em função da amplitude da rede duplamente periódica ou rede óptica simples [24]. Porém, não encontramos trabalhos teóricos que utilizam métodos variacionais que exploram o papel do coeficiente de não linearidade em função do potencial químico para o caso de uma rede óptica quase periódica. A aplicação de gaussianas como soluções de equações que descrevem o comportamento de gases aprisionados tem obtido sucesso em auxiliar pesquisadores nas interpretações de vários resultados experimentais [26]. É interessante ressaltarmos que alguns valores adotados em nosso trabalho, são provenientes de amplitudes de uma rede duplamente periódica ou rede simples $V_{0}=5[2,15]$, entretanto, outros valores podem ser utilizados.

\section{Conclusão}

Nós mostramos que a aproximação variacional é adequada para descrever e prever famílias de gap sólitons fundamentais formadas a partir do potencial de aprisionamento de uma rede óptica quase periódica em 1D para um Fermi superfluído. Como observado no caso para 1D, a aproximação variacional revelou que famílias de gap solitons são estáveis, mesmo variando a amplitude da rede quase periódica, entretanto, ultrapassam as bandas de Bloch. Um comportamento semelhante foi observado em um gás de bósons e férmions na Ref. [2,15,24].

Os resultados apresentados neste artigo abrem portas para a realização de futuros trabalhos, como o estudo teórico de gaps sólitons iluminados em outras dimensões confinados por potenciais de redes ópticas quase periódicas para um gás de férmions em um regime BSC; transição de gaps sólitons em um regime estável para instável através do movimento da rede óptica quase periódica; efeitos colisionais entre sólitons. O mesmo procedimento pode ser realizado experimentalmente, porém é necessária a introdução de tais potenciais de confinamentos.

O comportamento dos gaps sólitons iluminados obtidos ao variarmos as amplitudes da rede ópticas quase periódicas mostraram que eles são estáveis. Como a amplitude do potencial periódico de uma rede óptica quase periódico unidimensional depende de alguns parâmetros como a amplitude (Eq. 5), incentivamos o leitor realizar os cálculos variacionais para outros valores de amplitude da rede óptica.

\section{Referências}

[1] K. Huang, Statistical Mechanics (John Wiley and Sons Ltd., New York, 1988); S.R.A. Salinas, Introdução à Física Estatística (Editora Universidade de São Paulo, São Paulo, 2013), $2^{a}$ ed, $3^{a}$ reimp; S.R. Dahmen, Rev. Bras. Ensino Física 27, 283 (2005); B.F. Rizzuti e F.L. Forgerin, Rev. Bras. Ensino de Fisica 37, 1316 (2015).

[2] V.A. Nascimento, Rev. Bras. Ensino Física 33, 2305 (2011); V.A. Nascimento, C.L. Silva, A.V.D. Lanoa, Valdir A. Nascimento, A.F. Silva e P.C. Cassino, Rev. Bras. Ensino Física 36, 4314 (2014).

[3] G.P. Agrawal, Fiber-Optic Communications Systems (John Wiley \& Sons, Inc., New York, 2002), 3rd ed.; B.A. Malomed, Soliton Management in Periodic Systems (Springer Science + Busines Media, New York, 2006).

[4] J.V. Porto, S. Rolston, B.L. Tolra, C.J. Williams and W.D. Phillips, Phil. Trans. Math. Phys. Eng. Sci. 361, 1471 (2003); K.G.H. Vollbrecht, E. Solano and J.I. Cirac, Phys. Rev. Lett. 93, 220502 (2004).

[5] L.W. Cheuk, M.A. Nichols, M. Okan, T. Gersdorf, V.V. Ramasesh, W.S. Bakr, T. Lompe and M.W. Zwierlein, Phys. Rev. Lett. 114, 193001 (2015).

[6] W. Hofstetter, J.I. Cirac, P. Zoller, E. Demler and M.D. Lukin, Phys. Rev. Lett. 89, 220407 (2002).

[7] J.W. Park, S.A. Will and M.W. Zwierlein, Phys. Rev. Lett. 114, 205302 (2015).

[8] C. Groß, Nature Photonics 9, 482 (2015). 
[9] Q. Zwerger (ed) The BCS-BEC Crossover and the Unitary Fermi Gas, Vol. 836, (Springer, New York, 2011); M.W. Zwierlein, in: Novel Superfluids, Vol. 2, edited by K.H. Bennemann and J.B. Ketterson (Oxford University Press, Oxford, 2014).

[10] W.D. Phillips and H.J. Metcalf, Scientific American 256, 50 (1987).

[11] L. Khaykovich, F. Schreck, G. Ferrari, T. Bourdel, J. Cubizolles, L.D. Carr, Y. Castin and C. Salomon, Science 296, 1290 (2002); K.E. Strecker, G.B. Partridge, A.G. Truscott and R.G. Hulet, Nature 417, 150 (2002).

[12] S.L. Cornish, S.T. Thompson and C.E. Wieman, Phys. Rev. Lett. 96, 170401 (2006).

[13] M. Greiner, C.A. Regal and D.S. Jin, Nature 426, 537 (2003); M.W. Zwierleinn, C.A. Stan, C.H. Schunck, S.M.F. Raupach, S. Gupta, Z. Hadzibabic and W. Ketterle, Phys. Rev. Lett. 91, 250401 (2003).

[14] J.K. Chin, D.E. Miller, Y. Liu, C. Stan, W. Setiawan, C. Sanner, K. Xu, W. Ketterle, Nature 443, 961 (2006).

[15] B.A. Malomed, V.A. Nascimento and S.K. Adhikari, Mathematics and Computers in Simulation 80, 648 (2009). S.K. Adhikari and B.A. Malomed, Europhys. Lett. 79, 50003 (2007).

[16] R.B. Diener, G.A. Georgakis, J. Zhong, M. Raizen and Q. Niu, Phys. Rev. A 64, 033416 (2001).

[17] L. Guidoni, C. Triché, P. Verkerk and G. Grynberg, Phys. Rev. Lett 79, 3363 (1997).

[18] J.L. Cohen, B. Dubetsky and P.R. Berman, Phys. Rev. A 60, 3982 (1999).

[19] K. Huang and C.N Yang, Phys. Rev. A, 105, 767 (1957); N. Manin and L. Salasnich, Phys. Rev. A 71, 033625 (2005).

[20] A.L. Fetter and J.D. Walecka, Quantum Theory of Many Particle Systems (McGraw Hill, New York, 1971).

[21] P.J.Y. Louis, Elena A. Ostrovskaya and Y.S. Kivshar, Phys. Rev. A. 71, 023612 (2005); P.G. Kevrekidis and D.J. Frantzeskakis, Mod. Phys. Lett. B. 18, 173 (2004).

[22] V.M. Pérez Garcia, H. Michinel, J.I. Cirac, M. Lewenstein and P. Zoller, Phys. Rev. Lett. 77, 5320 (1996); V.M. Pérez Garcia, H. Michinel, J.I. Cirac, M. Lewenstein and P. Zoller, Phys. Rev. A. 56, 1424 (1997).

[23] T. Mayteevarunyoo and B.A. Malomed, Phys. Rev. A. 74, 033616, (2006); A. Gubeskys, B.A. Malomed and I.M. Merhasin, Etud. Appl. Math. 115, 255 (2005).

[24] P.J.Y. Louis, Elena A. Ostrovskaya and Y.S. Kivshar, Phys. Rev. A. 71, 023612 (2005); P.J.Y. Louis, E.A. Ostrovskaya, C.M. Savage and Y.S. Kivshar, Phys. Rev. A. 67, 013602 (2003).

[25] E.A. Ostrovskaya and Y.S. Kivshar, Phys. Rev. Lett. 90, 160407 (2003); E.A. Ostrovskaya, T.J. Alexan- der and Y.S. Kivshar, Phys. Rev. A. 74, 023605 (2006).

[26] J.H.V. Nguyen, P. Dyke, De Luo, B.A. Malomed and R.G. Hulet, Nature Physics 10, 918 (2014). 